\title{
Modulated Mutually Coupled Nano-Lasers
}

\author{
Han, Hong; Shore, K. Alan
}

\section{IEEE Journal of Quantum Electronics}

Published: 01/04/2017

Peer reviewed version

Cyswllt i'r cyhoeddiad / Link to publication

Dyfyniad o'r fersiwn a gyhoeddwyd / Citation for published version (APA):

Han, H., \& Shore, K. A. (2017). Modulated Mutually Coupled Nano-Lasers. IEEE Journal of Quantum Electronics, 53(2).

\footnotetext{
Hawliau Cyffredinol / General rights

Copyright and moral rights for the publications made accessible in the public portal are retained by the authors and/or other copyright owners and it is a condition of accessing publications that users recognise and abide by the legal requirements associated with these rights.

- Users may download and print one copy of any publication from the public portal for the purpose of private study or research.

- You may not further distribute the material or use it for any profit-making activity or commercial gain

- You may freely distribute the URL identifying the publication in the public portal ?
}

Take down policy

If you believe that this document breaches copyright please contact us providing details, and we will remove access to the work immediately and investigate your claim. 


\title{
Modulated Mutually-Coupled Nano-Lasers
}

\author{
Hong Han, K. Alan Shore, Senior Member, IEEE
}

\begin{abstract}
The dynamics of mutually coupled nano-lasers subject to direct current modulation has been analysed using rate equations which include the Purcell cavity-enhanced spontaneous emission factor $F$ and the spontaneous emission coupling factor $\beta$. Subject to two different modulation frequencies, the mutuallycoupled nano-lasers display two general types of response. The laser with the lower modulation frequency simply exhibits a response at that modulation frequency. This we term a zero crosstalk response. On the other hand, at higher modulation frequencies the system displays a variety of dynamical responses which, in addition to zero cross-talk, includes a range of behaviours which are classified from low cross-talk through to a complicated non-linear response. The precise behaviour being dependent on the depth of modulation and the laser bias currents. The operational significance of the zero cross-talk regime is that it permits access to a simple periodic response at the modulation frequency. With a view to utilisation, it is established that the region of zero cross-talk response enlarges with increasing modulation depth and increasing bias current. In this way conditions are established in which the lasers may act independently. The propensity for zero cross-talk response under stronger driving is consistent with previous analysis wherein modulated nano-lasers may have superior characteristics in the large-signal regime.
\end{abstract}

Index Terms-Mutually-coupled semiconductor lasers, nanolasers, enhanced spontaneous emission, high-frequency modulation

\section{INTRODUCTION}

$\mathrm{M}$ utually coupled lasers have been investigated for many decades [1]. Activity on mutually coupled semiconductor lasers also has long antecedents [2], [3] with significant effort having been given to identifying regimes of synchronization and instabilities [4]-[6]. In such work a variety of semiconductor lasers have been utilised with Vertical Cavity Surface Emitting Lasers (VCSELs) providing particularly rich dynamical scenarios [7]. Optical injection is well-known as a means for enhancing the modulation bandwidth of semiconductor lasers [8] and in recent work modulation bandwidth enhancement in mutually-coupled monolithically integrated laser diodes has been reported [9]. Semiconductor

Manuscript submitted November 30, 2016. The authors gratefully acknowledge the financial support provided in part by the Sêr Cymru National Research Network in Advanced Engineering and Materials, and in part by the International Science \& Technology Cooperation Program of China (2014DFA50870) and the National Natural Science Foundation of China (61527819).

H. Han is the School of Electronic Engineering, Bangor University, Wales, LL57 1UT, UK. and with the Taiyuan University of Technology, College of Physics and Optoelectronics, Key Laboratory of Advanced Transducers and Intelligent Control System, Ministry of Education, Taiyuan 030024, China.

K. Alan Shore is with the School of Electronic Engineering, Bangor University, Wales, LL571UT, UK.

(email: hanhong@tyut.edu.cn, k.a.shore@bangor.ac.uk) nano-lasers [10], [11] are of interest not least for their potential for inclusion in photonic integrated circuits.

In recent work we have initiated theoretical investigations of the dynamical behaviour of mutually coupled nano-lasers [12], [13]. In [12] effort was directed at the analysis of the behaviour of identical nano-lasers. There attention was given to the role played by the Purcell spontaneous emission enhancement factor $F$ and the spontaneous emission coupling factor $\beta$ with different distances, $D$, between the lasers and for a range of laser bias currents [12].

Subsequent work [13] sought to broaden the analysis of this system by detailing the dynamical behaviour of coupled nanolasers when operated under non-identical conditions and including effects arising due to frequency detuning between the lasers. That work, in particular, identified the presence of highfrequency oscillations (of order $100 \mathrm{GHz}$ ) which arose in several circumstances. That analysis allowed the delineation of significant dynamical features but did not exhaust all opportunities for influencing the dynamics of mutually coupled nano-lasers. It was explicitly recognised in [12] that further analysis should incorporate effects arising do to the mutual coupling of non-identical lasers which would thereby enable the definition of the dynamical regimes accessed by this system as has been previously performed in other configurations (see e.g. [14]). In the present paper consideration is given to the system of mutually coupled nano-lasers when one or both are subject to direct-current modulation.

The experimental context for this work is established by work performed on a variety of nano-laser structures such as, micro-post [15] nano-pillar and bowtie [16], [17], Fabry-Perot [18], nanowire [19], and nano-patch [20] lasers, where continuous wave lasing is observed by optical pumping and electrical pumping [21]. In early work, the impact of Purcell enhanced spontaneous emission on the modulation performance of nano-LEDs and nano-lasers [23] was examined. In addition to [12], [13], a number of recent investigations of the dynamical performance of nano-lasers have been undertaken. The behaviour of optically pumped nano-lasers has been studied including the role of the spontaneous emission factor, $\beta$, in achieving single mode operation of nano-lasers [23]. Ding et al. explored the dynamics of electrically pumped nano-lasers where the effects of $F$ and $\beta$ on nano-laser performance were studied [24]. A more recent investigation of the effect of $F$ and $\beta$ shows that modulation bandwidth of up to $60 \mathrm{GHz}$ can be achieved for metal clad nano-lasers [25]. Theoretical work has also been reported on the control of dynamical instability in such lasers [26].

Enhanced spontaneous emission, coupled with reduced laser threshold current, can lead to a reduction of the laser turn-on delay. Strong damping will give rise to a long tail in the switchoff dynamics of the laser and hence will compromise both analogue and digital direct current modulation of the laser. In recent work on the effect of external optical feedback in nano- 
lasers, it has been identified that strong damping of the relaxation oscillations due to high $F$ and $\beta$, causes the chaos to occur at higher feedback fractions [27]. Similar conclusions have been drawn in explorations of phase-conjugate optical feedback effects in nano-lasers [28]. Nano-lasers subject to external optical injection have also been predicted to exhibit more stable behaviour [28]. It was in this context that investigations of the dynamical behaviour of mutually coupled nano-lasers were initiated [12], [13]. The theme of the present paper is the impact of direct current modulation on the behaviour of mutually-coupled nano-lasers. A particular area of interest is their response to rather high modulation frequencies.

The paper is structured as follows. The nano-laser dynamical model is introduced in section II. Results given in section III delineate the main dynamical behaviour which arises when one nano-lasers are subject to modulation. Section IV aims to draw general conclusions concerning the stability properties of dual modulated mutually-coupled nano-lasers. Finally, in section V, conclusions are drawn based on the results obtained.

\section{NANO-LASER DYNAMICS}

A schematic diagram of modulated mutually coupled nanolasers is shown in Fig. 1. This system is modelled using modified forms of rate equations which incorporate the Purcell enhanced spontaneous emission factor, $F$ and spontaneous emission coupling factor, $\beta$ have been included as introduced in [21].

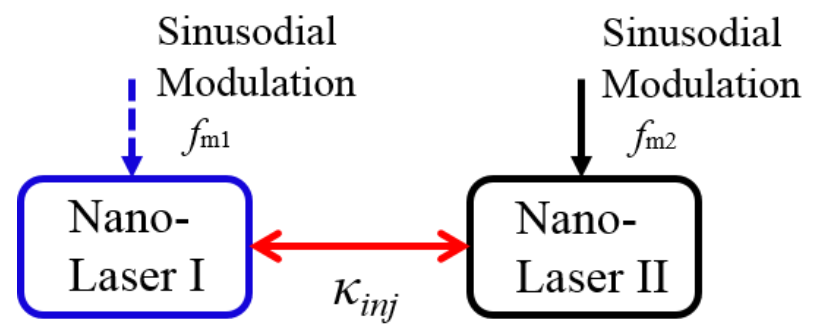

Fig. 1 Schematic diagram of modulated mutually-coupled semiconductor nanolasers.

Work by Gu. et al. [29] and Gerard et al. [30] has included the detailed calculation of the spontaneous emission rate in nano-lasers. This work has shown that there is an interdependence between the spontaneous emission coupling factor and the Purcell enhancement factor. Such an approach has been adopted by [14] in the formulation of dynamical equations for nano-lasers. However, the precise relationship between these two factors is dependent upon the specific nanolaser structure under consideration. In this context, and notwithstanding the work in [29], [30], the Purcell factor and the spontaneous emission factor are taken to be independent parameters. In this way it is possible to identify the trends in device performance consequent to changes in these two parameters. It is fully recognised, however, that in a practical context and due to the work of [29], [30], there will be constraints on the accessible values of these parameters. In the present work we choose one combination only of those parameters.

It is underlined that the Purcell factor and the spontaneous emission coupling factor impact the spontaneous emission rate as shown in Eqs. (1) and (2) below. Specifically it is pointed out that for Purcell factors greater than unity an effective reduction in the carrier lifetime will result. Similarly an increase of the spontaneous emission coupling factor towards unity also causes an effective reduction of the carrier lifetime. In contrast, the phase Eq. (3) is dependent on the laser gain and hence is not affected by the enhanced spontaneous emission.

$$
\begin{aligned}
& \frac{d S_{\mathrm{I}, \mathrm{II}}(t)}{d t}=\Gamma\left[\frac{F \beta N_{\mathrm{I}, \mathrm{II}}(t)}{\tau_{n}}+G_{n}\left(N_{\mathrm{I}, \mathrm{II}}(t)-N_{t h}\right) S_{\mathrm{I}, \mathrm{II}}(t)\right] \\
& +2 \frac{\kappa_{i n j}}{\tau_{i n}} \sqrt{S_{\mathrm{I}, \mathrm{II}}(t) S_{\mathrm{II}, \mathrm{I}}\left(t-\tau_{i n j}\right)} \cos \left(\theta_{\mathrm{I}, \mathrm{II}}(t)\right) \\
& \frac{d N_{\mathrm{I}, \mathrm{II}}(t)}{d t}=\frac{I_{\mathrm{IIII}}}{e V_{b}}-\frac{N_{\mathrm{L}, \mathrm{II}}(t)}{\tau_{n}}(F \beta+(1-\beta)) \\
& -G_{n}\left(N_{\mathrm{L}, \mathrm{II}}(t)-N_{0}\right) S_{\mathrm{I}, \mathrm{II}}(t) \\
& \frac{d \phi_{\mathrm{I}, \mathrm{II}}(t)}{d t}=\frac{\alpha}{2} \Gamma G_{n}\left(N_{\mathrm{I}, \mathrm{II}}(t)-N_{t h}\right) \pm \Delta \omega \\
& -\frac{\kappa_{i n j}}{\tau_{i n}} \sqrt{\frac{S_{\mathrm{III}}\left(t-\tau_{i n j}\right)}{S_{\mathrm{I}, \mathrm{II}}(t)}} \sin \left(\theta_{\mathrm{IIII}}(t)\right) \\
& \theta_{\mathrm{I}, \mathrm{II}}(t)= \pm \Delta \omega t+\omega_{\mathrm{III},} \tau_{i n j}+\phi_{\mathrm{IIII}}(t)-\phi_{\mathrm{II}, \mathrm{I}}\left(t-\tau_{i n j}\right) \\
& I_{\mathrm{I}, \mathrm{II}}(t)=I_{d c}\left[1+h_{m 1, m 2} \sin \left(2 \pi f_{m 1, m 2} t\right)\right]
\end{aligned}
$$

In the rate equations including the modulation the subscripts 'I' and 'II' represent laser I and laser II respectively. $S(t)$ is the photon density and $N(t)$ is the carrier density, $\varnothing(t)$ is the phase of the laser, $\theta(t)$ is the phase of injection laser. $\Gamma$ is the confinement factor; $\tau_{n}$ and $\tau_{p}$ are the radiative carrier lifetime and photon lifetime respectively. $G_{n}$ is the differential gain that takes into account the effect of group velocity, $N_{o}$ is the transparency carrier density, $\epsilon$ is the gain saturation factor and $\alpha$ is the linewidth enhancement factor. $I_{\mathrm{dc}}=j I_{t h}$ is the dc bias current, where $j$ is the normalized injection current; $I_{\text {th }}$ is the threshold current $\left(I_{t h}=(F \beta+(1-\beta)) N_{t h} e V_{a} / \tau_{n}\right), V_{a}$ is the volume of the active region $e$ is the electron charge and $N_{t h}$ $\left(N_{t h}=N_{o}+1 / \Gamma g_{n} \tau_{p}\right)$ is the threshold carrier density. $\Delta \omega$ is the angular frequency detuning between laser I and laser II. $\tau_{i n j}$ $=D / c$ is the injection delay, where $D$ is the distance between laser I and laser II, $c$ is the speed of light in free space. $\tau_{\text {in }}$ $=2 n L / c$ is the round-trip time in of the laser cavity, where $L$ is the cavity length and $n$ is group refractive index. The mutuallycoupled optical injection into the laser I and laser II is controlled by the injection fraction, $\kappa_{i n j}$, which is related to the injection parameter[31]. Sinusoidal direct current modulation of the lasers included in Eq. (2) is characterised by a modulation frequency, $f_{\mathrm{m} 1}$ or $f_{\mathrm{m} 2}$, for the laser I and laser II, and the corresponding depth of modulation are $h_{\mathrm{m} 1}$ and $h_{\mathrm{m} 2}$. The values of the nano-lasers device parameters used in the simulations are provided in Table I.

Attention is drawn to the fact that an increase of spontaneous emission via the Purcell factor, $F$ or the spontaneous emission coupling factor $\beta$ may lead to a change in the laser threshold current [22]. This has been taken into account in our previous analysis [13]. In the present work, use is made of just one combination of these parameters viz; Purcell factor, $F=14$ and spontaneous emission coupling factor, $\beta=0.1$. The remaining device parameters are also chosen to be the same for both lasers. 
TABLE I

NANO-LASER DEVICE PARAMETERS

\begin{tabular}{lccc}
\hline \hline Wavelength & $\lambda$ & $1591 \mathrm{~nm}$ & {$[10]$} \\
Cavity length & $L$ & $1.39 \mu \mathrm{s}$ & {$[10]$} \\
Volume of active region & $V_{a}$ & $3.96 \times 10^{-19} \mathrm{~m}^{3}$ & {$[10]$} \\
Group refractive index & $n$ & 3.4 & {$[10]$} \\
Round-trip time in inner cavity & $\tau_{\mathrm{in}}$ & $0.032 \mu \mathrm{s}$ & {$[10]$} \\
Photon lifetime & $\tau_{\mathrm{p}}$ & $0.36 \mathrm{ps}$ & {$[10]$} \\
Carrier lifetime & $\tau_{\mathrm{n}}$ & $1 \mathrm{~ns}$ & {$[32]$} \\
Differential gain & $G_{\mathrm{n}}$ & $1.65 \times 10^{-12} \mathrm{~m}^{-3} / \mathrm{s}$ & {$[10]$} \\
Mode confinement factor & $\Gamma$ & 0.645 & {$[10]$} \\
Line-width enhancement factor & $\alpha$ & 5 & {$[33]$} \\
Transparency carrier density & $N_{0}$ & $1.1 \times 10^{24} \mathrm{~m}^{-3}$ & {$[32]$} \\
Normalized injection current & $j$ & $2-10$ & \\
Modulation frequency & $f_{m}$ & $0-50 \mathrm{GHz}$ & \\
Modulation depth & $h_{m}$ & $0-0.8$ & \\
Coupling delay/ distance & $\tau_{\mathrm{inj}} / D$ & $0.05 \mathrm{~ns} / 0.015 \mathrm{~m}$ & \\
Cavity Purcell factor & $F$ & 14 & \\
Spontaneous emission coupling & $\beta$ & 0.1 & \\
Injection fraction & $\kappa_{\mathrm{inj}}$ & $0-0.6 \times 10^{-3}$ & \\
\hline \hline
\end{tabular}

It is noted that in recent work use has also been made of independent $F$ and $\beta$ parameters [34]. That work supported indications in [35] that, in some circumstances, microscopic modelling rather than rate equation analysis is needed to accurately capture dynamical features of nano-lasers. A specific recommendation of [34] is that nano-lasers should be operated in a regime where a combination of Purcell enhanced spontaneous emission and Rabi oscillations could provide modulation bandwidths of $350 \mathrm{GHz}$. The present work does not utilise device parameters allowing entry into that regime but nevertheless it is considered to be an exciting possibility which will stimulate further nano-laser device development.

\section{Single Frequency ModUlation DyNAMICAL BEHAVIOUR}

The aim of the paper is to progress appreciation of the dynamical behaviour of modulated mutually coupled nanolasers. In the first place, detailed attention is given to novel forms of dynamical response which arise when the lasers are modulated. In [13] due to the Purcell cavity-enhanced spontaneous emission factor $F$ and the spontaneous emission coupling factor $\beta$, mutually-coupled nano-lasers were shown to display strong stability when subjected to strong optical injection and high bias current. Thus, it may be anticipated that when subject to modulation, mutually coupled nano-lasers may display stability in the form of minimal interaction between the lasers. Also in [13] it was shown that high-frequency smallsignal oscillations could be established in unmodulated mutually coupled nano-lasers. It would be of interest to establish whether such oscillations can be enhanced by means of direct current modulation. In undertaking the present analysis, it has been assumed that relatively high frequencies of direct modulation - of order $50 \mathrm{GHz}$ - can be applied. This is rather less than the order $100 \mathrm{GHz}$ oscillations which were seen in [13] and hence enhanced modulation responses are not expected to be revealed here. Our caution in restricting the assumed modulation frequency acknowledges that $100 \mathrm{GHz}$ direct current modulation may not be routinely used in engineering contexts. But, in the expectation that advances will continue to be made in the relevant electronics, we are confident that exploration of this regime will become of practical relevance.

The results presented here have been found using the rate Eqs. (1)-(5). The bias current used to drive the lasers is an important operational parameter and dependences of behaviour on this parameter are discussed. Moreover the modulation frequency and depth of modulation are clearly salient parameters, as has been considered in the case of modulated stand-alone nano-lasers [25]. In the present paper, we also focus the attention on the various responses which may be elicited by changing these latter parameters.

\section{A. Coupling Strength Dependence}

Consideration is first given to a system of mutually-coupled nano-lasers when laser I is unmodulated but laser II is subject to $10 \mathrm{GHz}$ direct current modulation. Both lasers are assumed to have bias currents of $2 I_{\text {th. }}$.

Given the interest in mutual interactions a significant parameter is the coupling, $\kappa_{i n j}$, between the lasers. As the mutual coupling between the lasers increases, the photon density time series of the unmodulated laser I changes from quasi-periodicity to multiple -periods, as shown in blue in Figs. 2 to 4 . In contrast, as shown in black, for the modulated laser II, the output is transformed from a 'steady-state' with period-1 in Figs. 2 and 3 to steady-state of period-2 in Fig. 4. The spectra shown in these figures evidence the influence of strong coupling between the lasers whereby increased stability results in more stable output and hence cleaner spectra. Similar observations have been made in earlier work [31].
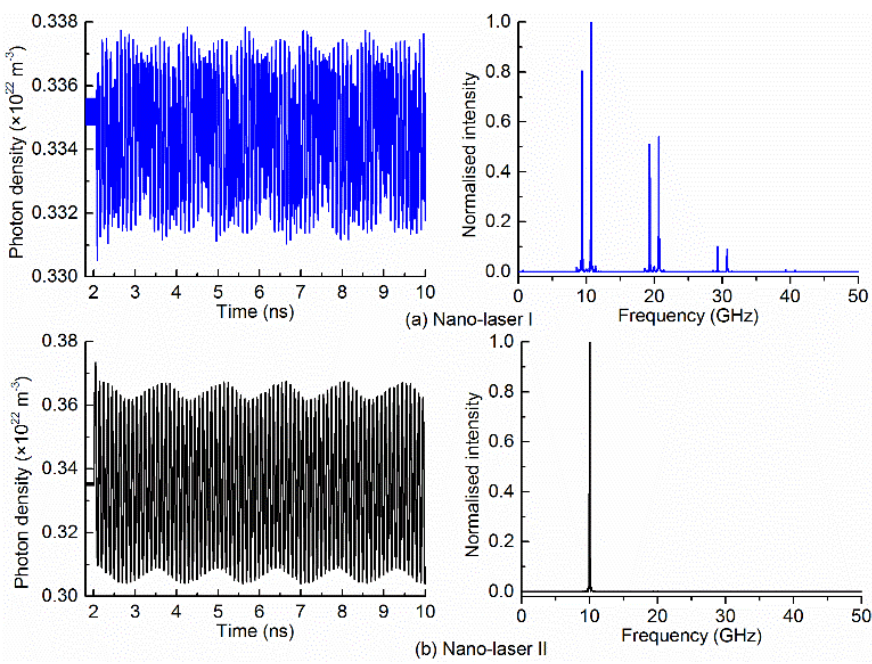

Fig. 2 Photon density time series and FFT with bias current $2 I_{\text {th }}$, at $\kappa_{i n j}=0.1 \times$ $10^{-3}$. (a) unmodulated nano-laser I (blue); (b) modulated nano-laser II (black) with $f_{\mathrm{m} 2}=10 \mathrm{GHz}$ and $h_{\mathrm{m} 2}=0.2$. 

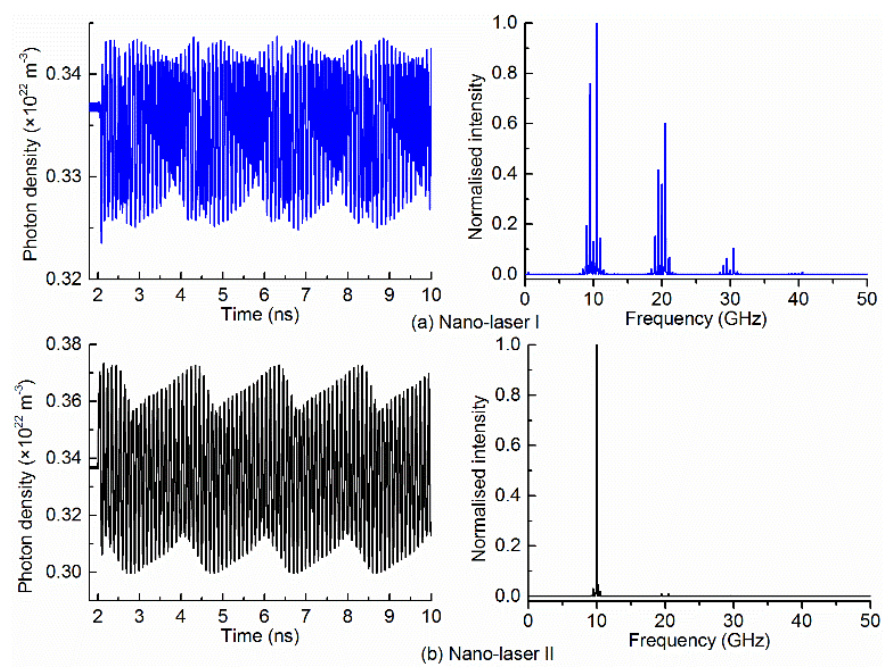

Fig. 3 Photon density time series and FFT with bias current $2 I_{\text {th }}$, at $\kappa_{i n j}=0.3 \times$ $10^{-3}$. (a) unmodulated nano-laser I (blue); (b) modulated nano-laser II (black) with $f_{\mathrm{m} 2}=10 \mathrm{GHz}$ and $h_{\mathrm{m} 2}=0.2$.
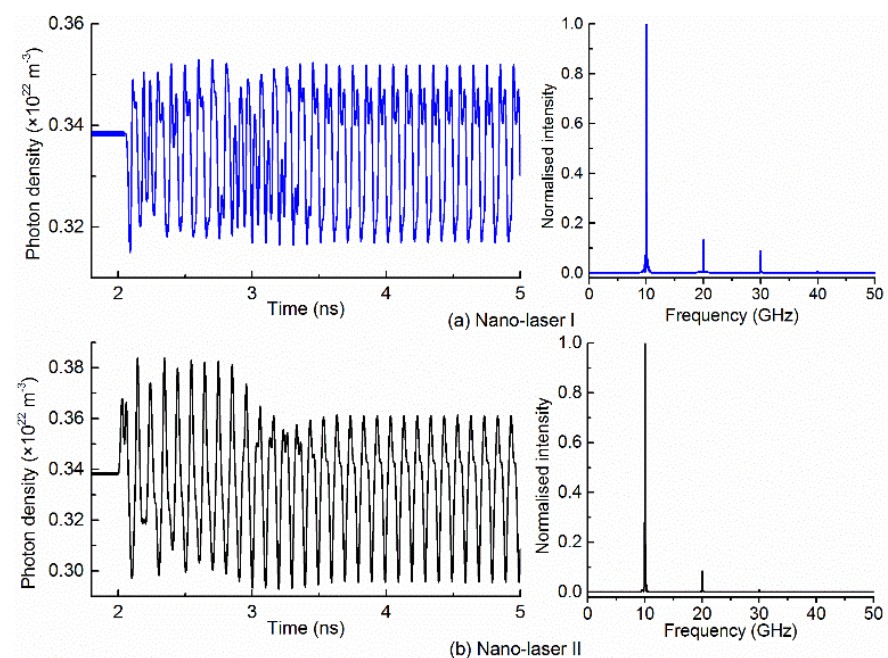

Fig. 4 Photon density time series and FFT with bias current $2 I_{\text {th }}$, at $\kappa_{i n j}=0.6 \times$ $10^{-3}$. (a) unmodulated nano-laser I (blue); (b) modulated nano-laser II (black) with $f_{\mathrm{m} 2}=10 \mathrm{GHz}$ and $h_{\mathrm{m} 2}=0.2$.

We find that increasing the modulation depth reduces the fluctuations of the photon density amplitude of the modulated nano-laser II. However, there is no change in the period of the photon density dynamics: that is the output of nano-laser II remains as period- 2 at $\kappa_{i n j}=0.6 \times 10^{-3}$. By the same token, when the modulation frequency is increased to say $20 \mathrm{GHz}$ whilst the coupling is maintained at $\kappa_{i n j}=0.6 \times 10^{-3}$, nano-laser II continues to exhibit a period-1 response.

\section{B. Bias current dependence}

The bias current dependence of the response is clearly of interest as has been shown previously $[12,13]$. In the present case, for a modulation frequency of $10 \mathrm{GHz}$, when we increase the bias current to $6 I_{\text {th }}$ the amplitude of the photon density of nano-laser II, as shown in black in Fig. 5, has obvious fluctuations at an injection coupling $\kappa_{i n j}=0.5 \times 10^{-3}$. Moreover, as shown in black in Fig. 6, this higher bias current enables the modulated nano-laser II to maintain period-1 output at the high injection coupling of $\kappa_{i n j}=0.6 \times 10^{-3}$ whilst the output of the unmodulated nano-laser I, shown in blue, has become multiperiodic.
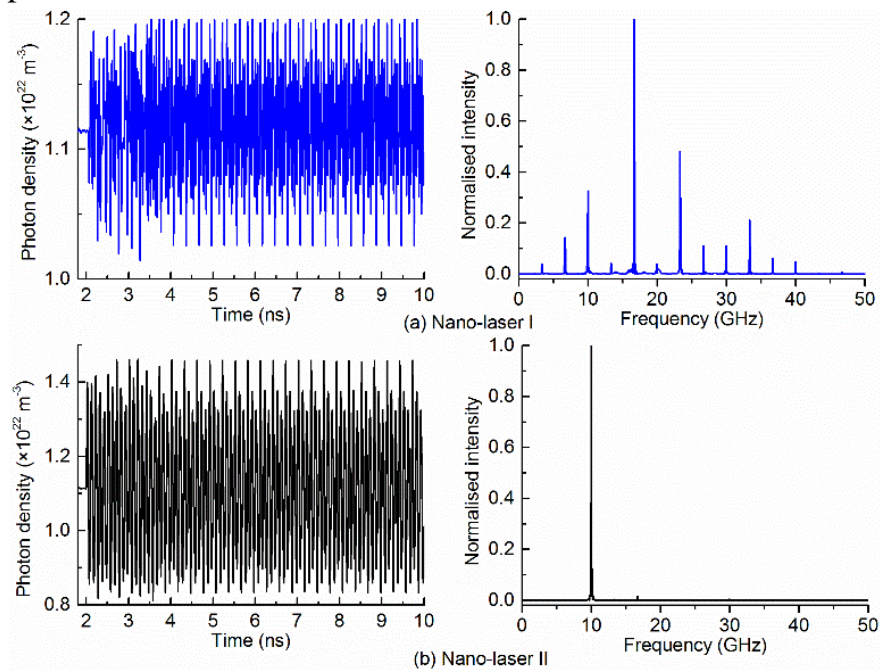

Fig. 5 Photon density time series and FFT with bias current $6 I_{\text {th }}$, at $\kappa_{\text {in }}=0.5 \times$ $10^{-3}$. (a) unmodulated nano-laser I (blue); (b) modulated nano-laser II(black) with $f_{\mathrm{m} 2}=10 \mathrm{GHz}$ and $h_{\mathrm{m} 2}=0.2$.
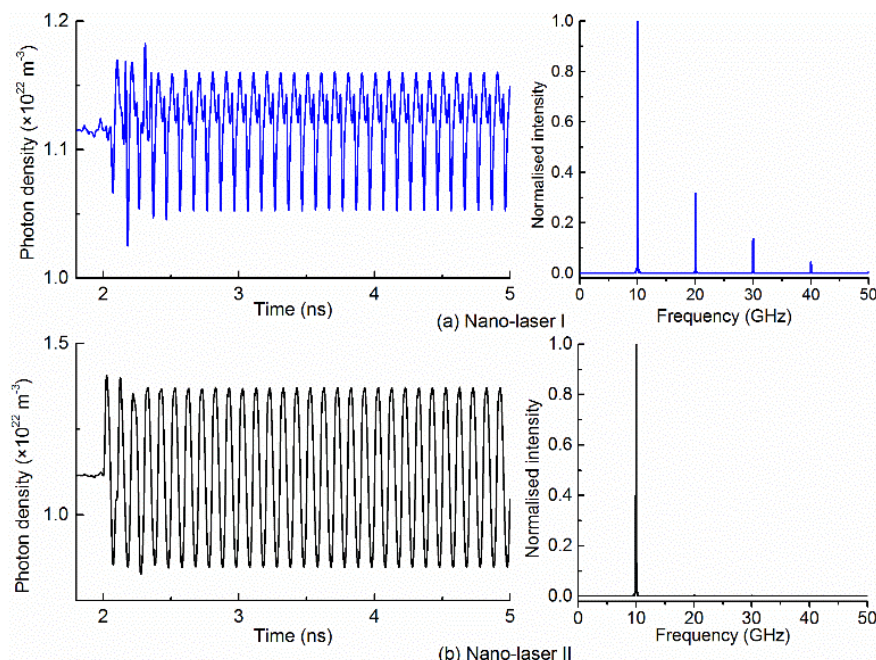

Fig. 6 Photon density time series and FFT with bias current $6 I_{\text {th }}$ at $\kappa_{\text {inj }}=0.6 \times$ $10^{-3}$. (a) unmodulated nano-laser I (blue); (b) modulated nano-laser II (black) with $f_{\mathrm{m} 2}=10 \mathrm{GHz}$ and $h_{\mathrm{m} 2}=0.2$.

Calculations, with a modulation frequency of $10 \mathrm{GHz}$ but with the yet much higher bias current of $10 I_{\text {th }}$, are displayed in Fig. 7. It can be seen in Fig.7, that, possibly after some initial transients, the unmodulated nano-laser (shown in Fig. 7(a)) still exhibits multi-periodic output, while the modulated nano-laser (shown in Fig. 7(b)) exhibits a steady period-1 response. 

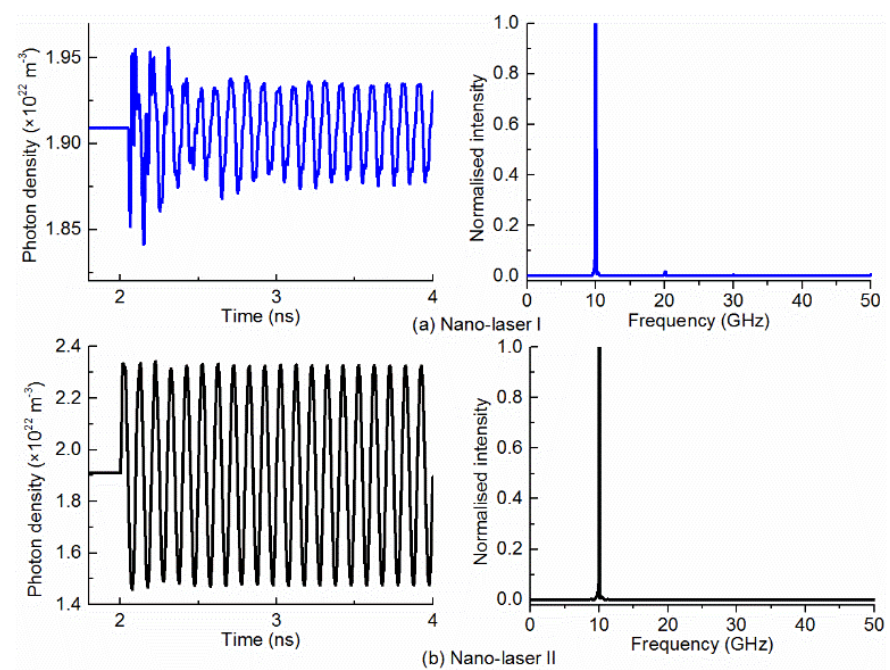

Fig. 7 Photon density time series and FFT with bias current $10 I_{\text {th }}$, at $\kappa_{i n j}=$ $0.6 \times 10^{-3}$. (a) unmodulated nano-laser I (blue); (b) modulated nano-laser II (black) with $f_{\mathrm{m} 2}=10 \mathrm{GHz}$ and $h_{\mathrm{m} 2}=0.2$.

\section{DUAL FREQUENCY MODULATION CROSS-TALK AND STABILITY PROPERTIES}

Having explored the response in the case of only one laser being modulated it is appropriate now to consider the interactions which may arise in this configuration when both nano-lasers are modulated.

In sub-section IVB general conclusions will be drawn on the overall response of nano-lasers to direct current modulation. As a basis for that, it is necessary to depict exemplar dynamical time series emerging from the dual frequency modulated mutually-coupled nano-lasers. It is shown that several species of relatively unusual dynamical behaviour can arise in favourable circumstances. In some cases, there is a need to examine the dynamics in some detail in order to discern the presence of those species. In turn, those forms of dynamics raise issues in terms of the classification of the overall behaviour of the system. Having presented the variety of dynamics we define, in sub-section IVA our nomenclature for the observed behaviour. That nomenclature underpins the results given in section IVB.

\section{A. Interpretation and classification of dynamics}

Having displayed representative examples of the dynamics appearing in one modulated mutually-coupled nano-lasers, attention is now given to interpreting and classifying the observed behaviour for dual modulation. An important issue is the extent to which the modulated behaviour of one laser affects that of the other. Such an interaction may, in general, be termed cross-talk. Here we classify the varieties of cross-talk which may arise in the situation under analysis.

In the case of both lasers being modulated, in order to make that cross-talk apparent, it will be assumed that the modulation frequencies are distinct. Moreover to avoid ambiguity due to the possible appearance of harmonics we choose non-integer multiples when selecting the modulation frequencies. In that spirit we first set a $10 \mathrm{GHz}$ modulation frequency for nano-laser II, and choose a modulation frequency of $25 \mathrm{GHz}$ for nano-laser I. Except for the modulation frequency, all the other parameters, such as bias current, injection coupling, depth of modulation are the same for both nano-lasers.

In the zero cross-talk state, there is no frequency component derived from one nano-laser which appears in the other nanolaser. It is pointed out that this behaviour does not need to be reciprocal. Figure 8 gives an example of zero cross-talk state for nano-laser I. It can be observed in Fig. 8(b) that the most prominent spectral feature is at the modulation frequency $(25$ $\mathrm{GHz}$ ). In addition to that the second harmonic, that is $50 \mathrm{GHz}$, can be observed in Fig.8 (b). Most importantly, however, there is no spectral component related to the $10 \mathrm{GHz}$ modulation frequency of nano-laser II. In this case it has also been found that no spectral component from nano-laser I appears in the spectrum nano-laser II. As such, the zero cross- talk here is reciprocal.
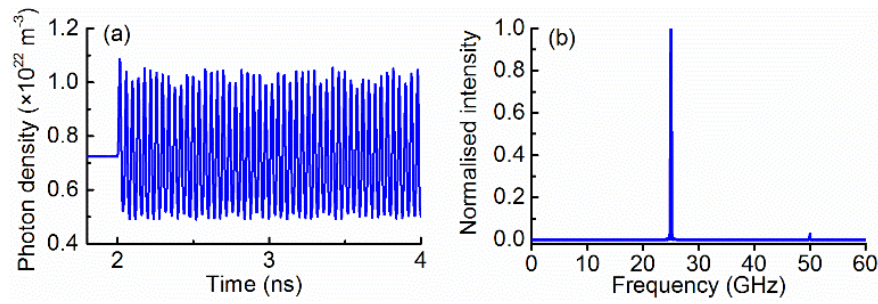

Fig. 8 Photon density time series (a) and FFT (b) of nanolaser-I with bias current $4 I_{\text {th }}$, at $\kappa_{i n j}=0.3 \times 10^{-3}, f_{\mathrm{m} 1}=25 \mathrm{GHz}, f_{\mathrm{m} 2}=10 \mathrm{GHz}, h_{\mathrm{m} 1}=h_{\mathrm{m} 2}=0.6$.

Changes in the modulation frequency and/or the depth of modulation can eradicate such zero cross-talk. In these cases spectral signatures of the modulation of one laser become apparent in the other. As the relative strengths of these signatures may vary significantly we identify three regimes of cross-talk viz: low cross-talk, medium cross-talk and strong cross-talk. For the low cross-talk the relative strengths ratio is below 0.5 , the medium one is between 0.5 and 1 , the strong one is equal or above 1. Examples of these are shown in Fig. 9 (a), (b) and (c), respectively for nano-laser I which always has a higher modulation frequency than nano-laser II.
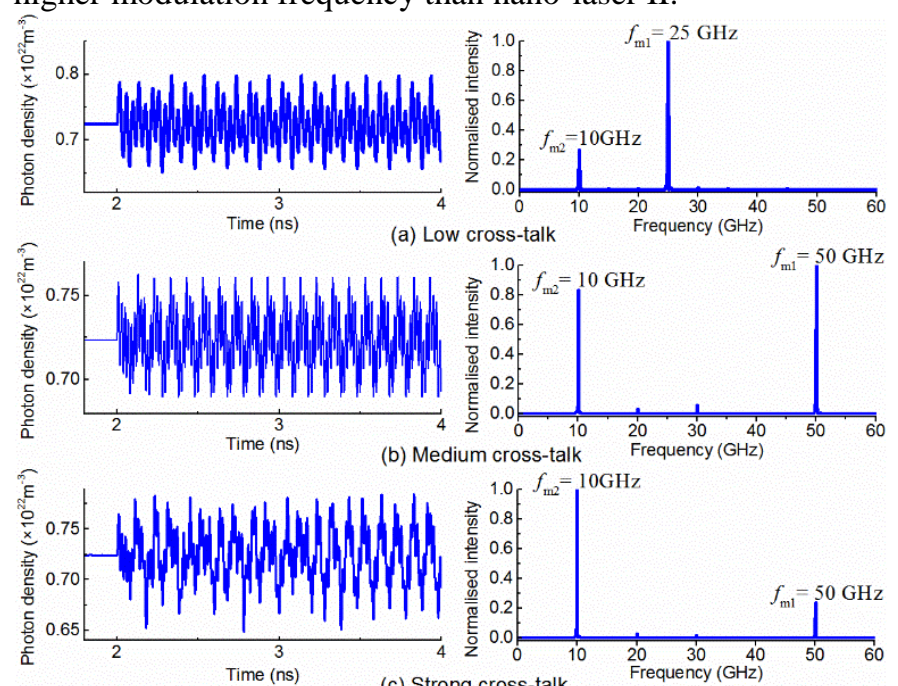

(c) Strong cross-talk

Fig. 9 Photon density time series and FFT of nano-laser I: (a) low cross-talk with $4 I_{\text {th }}$ at $\kappa_{i n j}=0.3 \times 10^{-3}, f_{\mathrm{m} 1}=25 \mathrm{GHz}, f_{\mathrm{m} 2}=10 \mathrm{GHz}$ and $h_{\mathrm{m} 1}=h_{\mathrm{m} 2}=0.1$; (b) medium cross-talk with $4 I_{\text {th }}$ at $\kappa_{i n j}=0.2 \times 10^{-3}, f_{\mathrm{m} 1}=50 \mathrm{GHz}, f_{\mathrm{m} 2}=10 \mathrm{GHz}$ and $h_{\mathrm{ml}}=h_{\mathrm{m} 2}=0.1$; (c) strong cross-talk with $4 I_{\mathrm{th}}$ at $\kappa_{i n j}=0.5 \times 10^{-3}, f_{\mathrm{m} 1}=50 \mathrm{GHz}$, $f_{\mathrm{m} 2}=10 \mathrm{GHz}$ and $h_{\mathrm{m} 1}=h_{\mathrm{m} 2}=0.1$. 
The calculations show further that nano-laser II exhibits no signature of the modulation of nano-laser I. In fact the output of nano-laser II is either steady-state period-1 or period-2 depending on the injection coupling and modulation depth. This provides an example where the classified cross-talk behaviour is not-reciprocal. That is, one laser displays the signature of the modulation of the other but not vice versa.

In addition to the various forms of cross-talk defined above, other more complicated responses can arise. Such behaviour we term a non-linear response. An example of such a nonlinear response is displayed in Fig. 10 for nano-laser I modulated at $50 \mathrm{GHz}$. In Fig.10 (b) it is seen, as expected, that the most prominent spectral feature is at the modulation, frequency. However additional clusters of frequencies are apparent around $10 \mathrm{GHz}$ and $20 \mathrm{GHz}$-harmonics of the modulation frequency of nano-laser II. These clusters of frequencies are unlike the cross-talk affected spectra shown in Fig.9. It is recalled that such a non-linear response also occurs in the single modulated mutually-coupled nano-lasers considered in section III. Cluster frequencies from the modulated nano-laser II are observed in FFT of nano-laser I as shown in Figs. 2 and 3. However, this non-linear response does not appear in the nano-laser I whose modulation frequency is low compared with that of nano-laser II. Once again this behaviour is non-reciprocal: the output of nano-laser II maintains steady-state period-1 or period-2 behaviour at its modulation frequency, independent of the behaviour of nano-laser I - that is zero cross-talk response. In the calculations performed for this work, it appears to be a universal feature that the nano-laser subject to the lower modulation frequency exhibits zero cross-talk.

The underlying physical reason for this is that the excursion of the photon density of the laser subject to higher modulation frequency is small [25]. As such the light coupled to the laser subject to the lower modulation frequency has a negligible impact on the laser subject to the lower modulation frequency.

In this situation in endeavouring to classify the behaviour of dually modulated mutually-coupled nano-lasers, attention may be focussed on the behaviour of the nano-laser subject to the higher modulation frequency. This approach is adopted in the next sub-section.
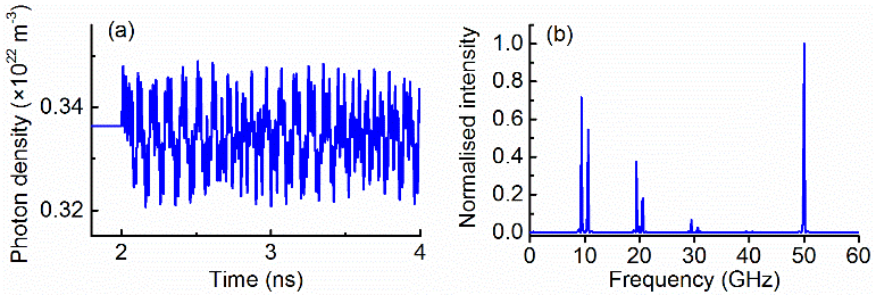

Fig. 10 Photon density time series (a) and FFT (b) of nano-laser I with bias current $2 I_{\text {th }}$, at $\kappa_{i n j}=0.3 \times 10^{-3}, f_{\mathrm{m} 1}=50 \mathrm{GHz}, f_{\mathrm{m} 2}=10 \mathrm{GHz}, h_{\mathrm{m} 1}=h_{\mathrm{m} 2}=0.2$.

\section{B. Modulation Response Regimes}

On the basis of the classification detailed in the previous subsection, attention is now given to categorising the behaviour of the nano-laser subject to the higher modulation frequency here nano-laser I. The primary operational parameters which are utilised for this purpose are the depth of modulation and the strength of coupling between the lasers. Attention will be given to how the response of the system changes with the increased bias current and with increases in the modulation frequency of nano-laser I. In all cases it is assumed that nano-laser II is modulated at $10 \mathrm{GHz}$.

Our previous work [13] had indicated that mutually-coupled nano-lasers exhibit strong stability when subjected to strong optical injection and high bias current. Thus, as we expected, when subject to modulation, mutually coupled nano-lasers display stability in the form of zero cross-talk response. The responses of nano-laser I are shown in Fig.11 (a) and (b), where the bias current is $2 I_{\mathrm{th}}$ and $4 I_{\mathrm{th}}$, respectively. It is observed that, with strong injection coupling, nano-laser I maintains a zero cross-talk response $(\bullet)$ even for large modulation depths $\left(h_{\mathrm{m}}=0.6\right.$ and $h_{\mathrm{m}}=0.8$ ) as shown in Fig. 11(a). This tendency is confirmed by Fig.11(b), where with increased bias current the zero cross-talk regions are significantly enlarged.

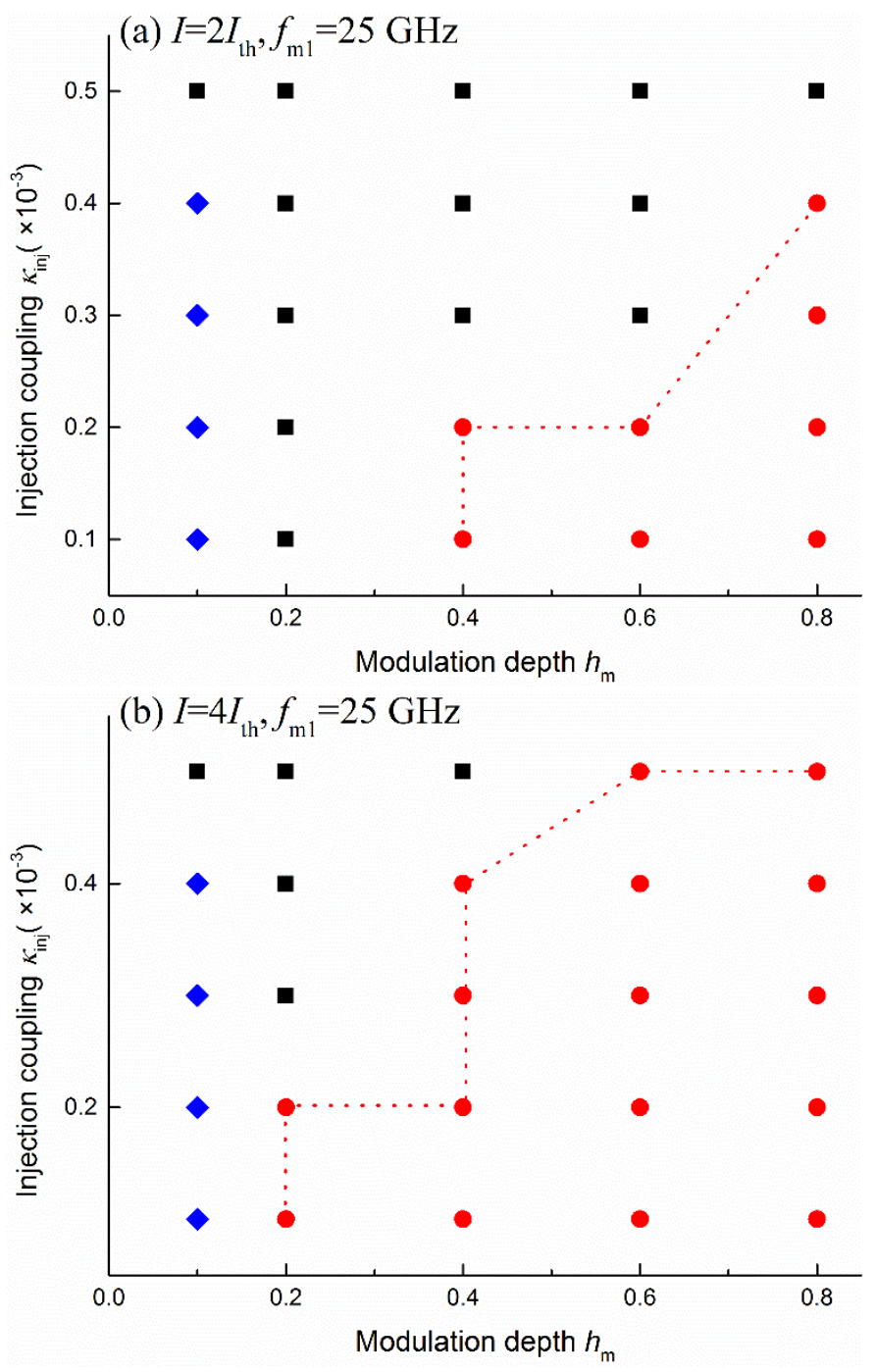

Fig. 11 Regions for different classification dynamics, over a range of modulation depth and injection coupling. Zero cross-talk (๑), non-linear response( $\mathbf{\square})$ and low cross-talk( $)$ region of the nano-laser I under $25 \mathrm{GHz}$ modulated frequency whilst nano-laser II with $f_{\mathrm{m} 2} 10 \mathrm{GHz}$.

In order to reveal modulation frequency effects, for the results of Fig. 12, we increase the modulation frequency of nano-laser I to 50GHz. Comparing Fig. 12(a)-(b) with Figs. 11(a)-(b), it is observed that increased modulation frequency enhances cross-talk effects, to include non-linear ( $\boldsymbol{\square})$, low 
cross-talk ( $\bullet$ ), medium cross-talk $(\boldsymbol{\Delta})$ and strong cross-talk ( $\star$ ) responses. Consequently the zero cross-talk region $(\bullet$ )is visibly reduced in size in Fig. 12(a). However, the influence of the bias current is confirmed so that as bias current increases from $2 I_{\text {th }}$ to $6 I_{\text {th }}$, as in Figs. 12(a) to 12(c), the zero cross-talk region is steadily enlarged.

Here we focus on the dynamics of mutually-coupled nanolasers under different modulation frequencies. The combinations of modulation frequencies chosen $\left(f_{m 1}, f_{m 2}\right)$ include values within and beyond the $3-\mathrm{dB}$ bandwidth of the nano-laser. More details of the modulation response dependence on bias currents are given in [36].
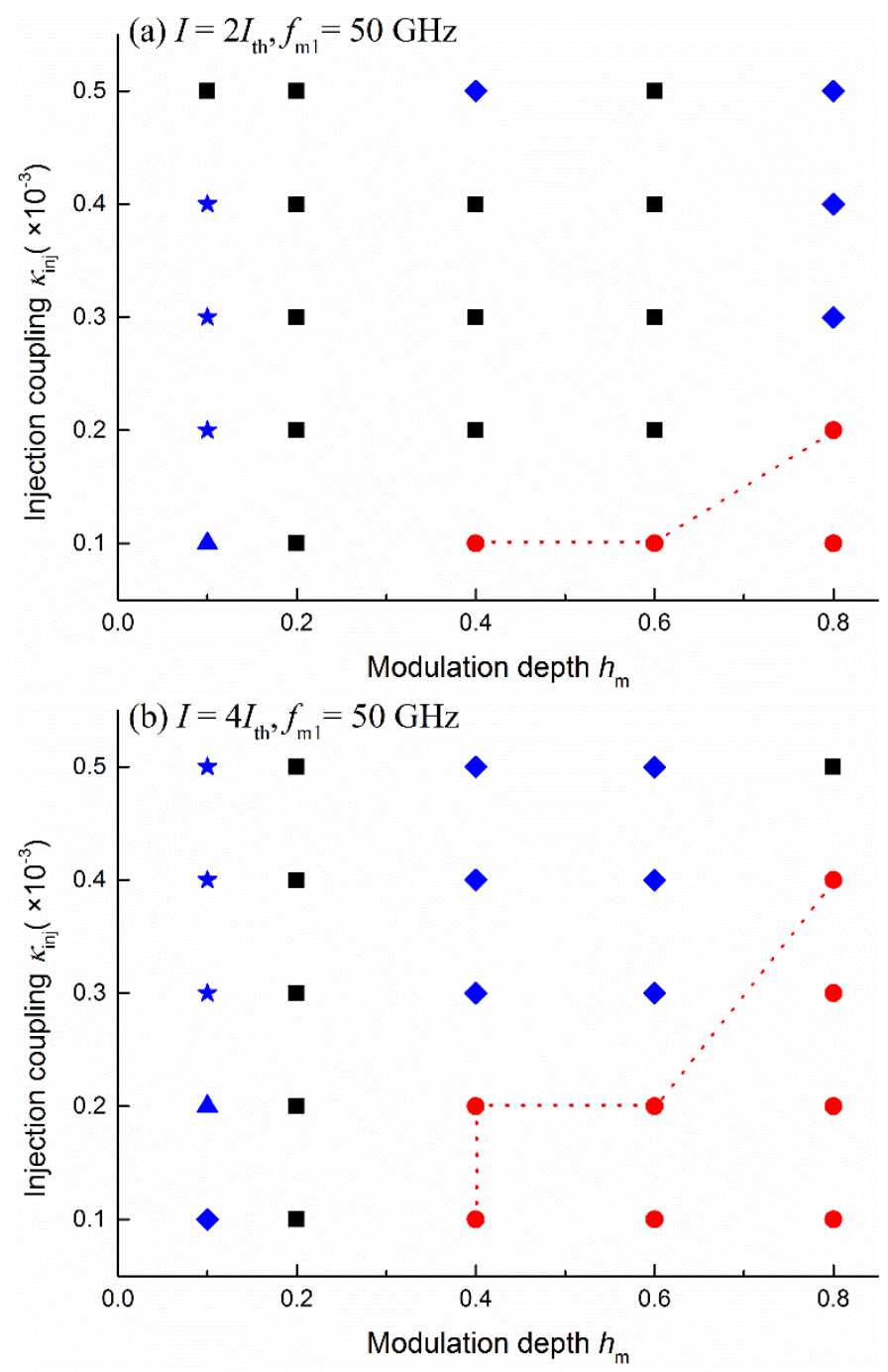

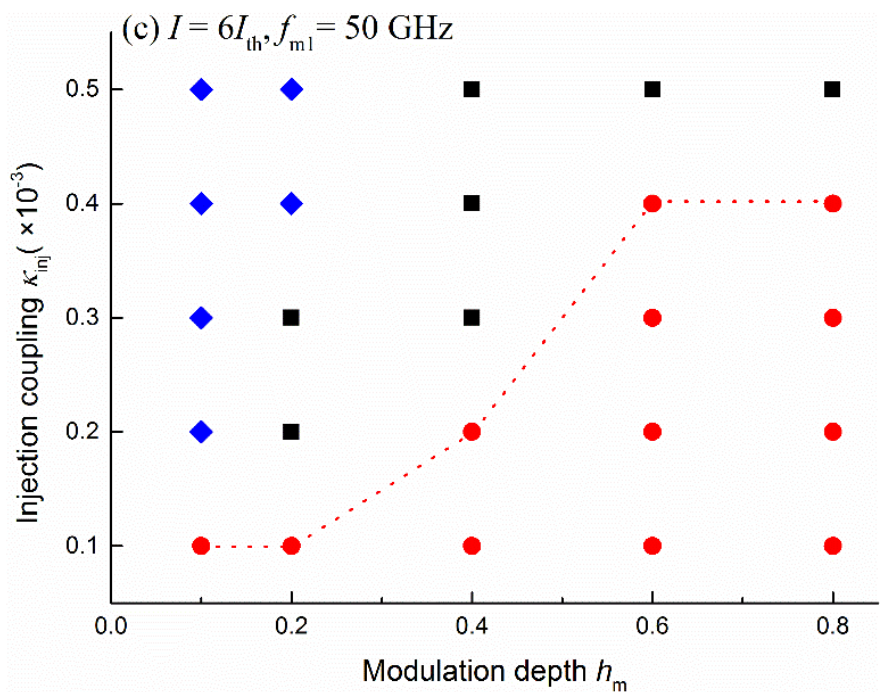

Fig. 12 Regions for different classification dynamics, over a range of modulation depth and injection coupling. Zero cross-talk $(\bullet)$, non-linear response ( $\mathbf{\square})$, low cross-talk $(\bullet)$, medium cross-talk ( $(\boldsymbol{\Lambda})$ and strong cross-talk $(\star)$ region of the nano-laser I under $50 \mathrm{GHz}$ modulated frequency whilst nanolaser II with $f_{\mathrm{m} 2} 10 \mathrm{GHz}$.

\section{CONCLUSIONS}

Theoretical analysis undertaken in this work shows that both single and dual modulated mutually coupled nano-lasers can give rise to a wide variety of dynamics. In the case of dual modulated mutually coupled nano-lasers, the interaction of the nano-lasers can range from zero cross-talk through to a complicated non-linear response. The actual response being the laser significantly affected by bias currents, injection coupling and the modulation depth. We find that at a relatively high modulation frequency, here $50 \mathrm{GHz}$, the modulated mutuallycoupled nano-lasers under high bias current display strong robustness with a large zero cross-talk region. In this region, the modulated mutually-coupled nanolasers act independently of each other. In particular, in this regime the response of the lasers is simply at the modulation frequency of the individual laser ( or possibly at a harmonic of that frequency). The facility to individually address a given nano-lasers without affecting the behaviour of the other mutually-coupled nano-lasers should find ready applications.

\section{REFERENCES}

[1] M. B. Spencer and W. E. Lamb, "Theory of two coupled lasers", Phys. Rev. A, vol. 5, no. 2, pp. 893-896, Feb. 1972.

[2] A. Hohl, A. Gavriledes, T. Ereux and V.Kovanis, " Localized synchronization for two delay-coupled semiconductor lasers", Phys. Rev. Letts, vol. 78, pp. 4745-4748, 1997.

[3] A. Hohl, A. Gavriledes,T. Erneux and V. Kovanis, "Quasiperiodic synchroniszation for two delay-coupled semiconductor lasers, Phys. Rev. A, vol. 59, p. 3941, 1999.

[4] J. Mulet, C. Mirasso, T. Heil and I. Fisher, "Synchronization scenario of two distant mutually coupled semiconductor lasers", J. Optics B: Quantum and semiclassical Optics, vol. 6, pp. 97-105, 2004.

[5] S. Yanchuk, K. R. Schneider and L. Recke, "Dynamics of two mutually coupled semiconductor lasers: instantaneous coupling limit", Phys. Rev. E, zuijvol. 69, p. 056221, 2004.

[6] P. Kumar, A. Prasad and R. Ghosh, "Strange bifurcation and phase-locked dynamics in mutually coupled diode laser systems", J. Phys. B: At. Mol . Opt. Phys., vol. 42, no. 14, pp. 145401-145407, 2009.

[7] Y. Hong, "Flat broadband chaos in mutually coupled VCSELS", IEEE J. Sel. Topics in Quant. Electron., vol.21, no. 6, pp. 1801007, Dec. 2015.

[8] E. K. Lau, L. H. Wong and M. C. Wu, "Enhanced modulation characteristics 
of optical injection-locked lasers: A tutorial", IEEE J. Sel. Top. Quantum Electron., vol. 15, no. 3, pp. 618-633, Apr. 2009.

[9] C. Sun, D. Liu, B. Xiong, Y. Luo, J. Wang, Z. Hao, Y. Han, L. Wang and $\mathrm{H}$. Li, "Modulation charecteristics enhancement of monolithically Integrated laser diodes under mutual injection locking", IEEE J. Selec. Topics in Quantum Electron., vol. 21, no. 6, p. 1802008, Dec. 2015.

[10] K. Ding, M. T. Hill, Z. C. Liu, L. J. Yin, P. J. V. Veldhoven and C. Z. Ning, "Record performance of electrical injection subwavelength metallic-cavity semiconductor lasers at room temperature", Opt. Express., vol. 21, no. 4, pp. 4728-4733, Feb. 2013.

[11] A. V. Maslov and C. Z. Ning, "Size reduction of a semiconductor nanowire laser by using metal coating", Proc. SPIE., vol. 6468, p. 646801, 2007.

[12] H. Han and K. A. Shore, "Dynamics and stability of mutually coupled nano-lasers", IEEE J. Quant. Electron., vol. 52, no. 11, 2000306, Nov. 2016.

[13] H. Han and K. A. Shore, "Noise-robust high-frequency small-signal oscillations in mutually-coupled nano-lasers", IEEE J. Lightw. Technol., submitted for publication 2016 .

[14] P. Kumar, A. Prasad and R. Ghosh, "Stable phase-locking of an external cavity diode laser subjected to external optical injection", J. Phys. B: At. Mol. Opt. Phys., vol. 41, p. 135402, Jun. 2008.

[15] M. Pelton, J. Vuckoviá, G. Solomon, C. Santori, B. Zhang, J. Plant and Y. Yamamoto, "Efficient source photons: a signle quantum dot in a micropost microcavity", Phys. E, vol. 17, pp. 564-567, Apr. 2003.

[16] S. Reitzensteinm, T. Heindel, C. Kistner,A. Rahimi-Iman, C. Schneider, S. Höfling and A. Forchel, "Low threshold electrically pumped quantum dotmicropillar lasers", Appl. Phys. Letts. vol. 93, no. 6, p. 061104, Aug. 2008.

[17] S. W. Chang, C. Y. A. Ni, and S. L. Chuang, "Theory for bowtie plasmonic nanolasers", Opt. Exp., vol. 16, no. 14, pp. 10580-10595, Jul. 2008.

[18] M. T. Hill, M. Marell, E. S. P. Leong, B. Smalbrugge, Y. Zhu, M. Sun, P. J. V. Weldhoven, E. J. Geluk, F. Karouta, Y. S. Oei, R. Notzel, C. Z. Ning and M.K. Smith, "Lasing in metal-insulator-metal sub- wavelength plasmonics waveguides", Opt. Exp., vol. 17, no. 13, pp. 11107-11112, Jun. 2009.

[19] R. F. Oulton, V. J. Sorger, T. Zentgraf, R. M. Ma, C. Gladden, L. Dai, G. Bartal and X. Zhang, "Plasmon lasers at deep subwavelength scale", Nature, vol. 461, pp. 629-632, Oct. 2009.

[20] K. Yu, A. Lakhani and M. C. Wu, "Subwavelength metal-optic semiconductor nanopatch lasers", Opt. Express., vol.18, no. 9, pp. 87908799, Apr. 2010

[21] K. Ding, Z. Liu, L. Yin, H. Wang, R. Liu, M. T. Hill, M. J. H. Marell, P. J.V. Veldhoven, R. Notzel and C. Z. Ning, "Electrical injection, continuous wave operation of sub wavelength-metallic-cavity lasers at 260 K”, Appl. Phys. Lett., vol. 98, no. 23, p. 231108, Jun. 2011.

[22] T. Suhr, N. Gregerson, Y. Yvind and J. Mork, "Modulation response of nanoLEDs and nanolasers exploiting Purcell enhanced spontaneous emission", Opt. Exp., vol. 18, no. 11, pp. 11230-11241, May. 2010.

[23] H. Gao, A. Fu, S. C. Andrews and P. Yang, "Cleaved-coupled nanowire lasers," Proc. Natl. Acad. Sci., vol. 110, no. 3, pp. 865-869, Jan. 2013.

[24] K. Ding and C. Z. Ning, "Metallic sub-wavelength-cavity semiconductor nanolasers", Light: Sci. Appl., vol. 1, no. 7, p. 20, Jul. 2012.

[25] Z. A. Sattar and K. A. Shore, "Analysis of the direct modulation response of nanowire lasers", IEEE. J. Lightw. Technol., vol. 33, no. 14 , pp. $3028-3033$, Jul. 2015.

[26] P. Kumar, and F. Grillot, "Control of dynamical instability in semiconductor quantum nanostructures diode lasers: role of phaseamplitude coupling”, Eur. Phys. J. Special Topics, vol. 222, no. 3-4, pp. 813-820, Jul. 2013

[27] Z. A. Sattar and K. A. Shore, "External optical feedback effects in semiconductor nano-lasers", J. Sel. Top. In Quant. Electron., vol. 21, no. 6, p. 1800106, Dec. 2015.

[28] Z. A. Sattar, and K. A. Shore, "Phase conjugate feedback effects on nano-lasers", IEEE J. Quant. Electron., vol. 52, no. 4, p. 1100108, Apr. 2016.

[29] Q. Gu, B. Slutsky, F. Vallini, J. S. T. Smalley, M. P. Nezhad, N. C. Frateschi and Y. Fainman, "Purcell effect in sub-wavelength semiconductor lasers", Opt. Express., vol. 21, no. 13, pp. 15603-15617, Jun. 2013.

[30] J. M. Gerard and B. Gayral, "InAs quantum dots: artificial atoms for solidstate cavity-quantum electrodynamics", Physica E., vol. 9, no. 1, pp. 131139, Jan. 2001.

[31] Z. A. Sattar and K. A. Shore, "Optical Injection Effects in Nano-lasers," IEEE J. Quant. Electron., vol. 52, no. 2, p. 1200108, Feb., 2016.

[32] L. A. Coldren and S. W. Corzine, Diode Lasers and Photonic Integrated Circuits. New York, NY, USA: Wiley, 1995.
[33] S. W. Chang, "Dressed linewidth enhancement factors in small semiconductor lasers", Opt. Exp., vol. 20, no. 15, pp. 16450-16470, Jul. 2012.

[34] R. Aust, T. Kaul, C-Z.Nong, B. Lignau and K.Ludge, "Modulation response of nano-lasers: what rate equation approaches miss", Opt and Quant. Electron., vol. 48 , 109, pp1-8, 2016.

[35] M. Lorke, T. Suhr, N. Gregersen and J. Mork, "Theory of nano-laser devices: rate equation analysis versus microscopic theory", Phys. Rev. B, vol. 87, 205310, 2013

[36] H. Han and K. A. Shore, "Zero cross-talk regime direct modulation of mutually-coupled nano-lasers", IEEE Photon. Technol. Lett., submitted for publication 2017.

Hong Han received the B.S. degree in physics from the University of Jiamusi, Jiamusi, China, and the Ph.D. degree from Harbin Institute of Technology, Harbin, China.

She is a Lecturer at the Taiyuan University of Technology and is currently a post- doctoral researcher at Bangor University. Her research work is in dynamic features of chaotic lasers and chaotic security communication. She is a member of OSA.

K. Alan Shore (SM'95) received the degree in mathematics from the University of Oxford, Oxford, U.K., and the Ph.D. degree from University College, Cardiff, Wales, UK.

He was a Lecturer at the University of Liverpool in 1979-1983 and then at the University of Bath where he became a Senior Lecturer in 1986, a Reader in 1990, and a Professor in 1995. He was a Visiting Researcher at the Center for High Technology Materials, University of New Mexico, Albuquerque, USA, in 1987. In 1989, he was a Visiting Researcher at the Huygens Laboratory, Leiden University, Netherlands. During the summers of 1990 and 1991, he was with the Teledanmark Research Laboratory and the MIDIT Center of the Technical University of Denmark, Lyngby. He was a Guest Researcher at the Electrotechnical Laboratory Tsukuba, Japan, in 1991. In 1992, he was a Visiting Professor at the Department of Physics, University de les IllesBalears, PalmaMallorca, Spain. He was a Visiting Lecturer in the Instituto de Fisica de Cantabria, Santander, Spain, in June 1996 and 1998, and a Visiting Researcher in the Department of Physics, Macquarie University, Sydney, Australia, in 1996, 1998, 2000, 2002, 2005, and 2008. In July/August 2001, he was a Visiting Researcher at the ATR Adaptive Communications Laboratories, Kyoto, Japan. In 1995, he was appointed to the chair of Electronic Engineering at Bangor University where he has been the Head of the School of Informatics and of the College of Physical and Applied Sciences. Between 2001 and 2008, he was the Director of "Industrial and Commercial Optoelectronics," a Welsh Development Agency Centre of Excellence. He was the Chair of the Welsh Optoelectronics Forum from 2006 to 2008 and has chaired the Photonics Academy for Wales, since its establishment in 2005. From 2008 to 2011, he was the Chair of the Quantum Electronics Commission of the International Union of Pure and Applied Physics. His research work has been principally in the area of semiconductor optoelectronic device design and experimental characterization with particular emphasis on nonlinearities in laser diodes and semiconductor optical waveguides. He has authored or co-authored more than 1000contributions to archival journals, books, and technical conferences. With Prof. D. Kane he co-edited the research monograph Unlocking Dynamical Diversity. His current research interests include the dynamics of vertical-cavity semiconductor lasers, applications of nonlinear dynamics in semiconductor lasers, and the design of nano-spin semiconductor lasers.

Prof. Shore cofounded and from 1987 to 2012 acted as the Organizer and Program Committee Chair for the International Conference on Semiconductor and Integrated Optoelectronics, which is held annually in Cardiff, Wales, UK. He has been a Program Member for several OSA conferences and was a Coorganizer of a Rank Prize Symposium on Nonlinear Dynamics in Lasers held at the Lake District, U.K., in August 2002. He chaired the Education and Training in Optics and Photonics conference held at the Technium OpTIC, Wales, 2009. He received the Royal Society Travel Grant to visit universities and laboratories in Japan in July 1988. From July to December 2010, he held a Japan Society for the Promotion of Science Invitation Fellowship in the Ultrafast Photonics Group, Graduate School of Materials Science, Nara Institute of Science and Technology, Nara, Japan. He is a Fellow of the Optical Society of America, the Institute of Physics, and the Learned Society of Wales for which he has served as a Council Member (2012-2015 and 2016-2019). 